\title{
Thermal compensation for large volume metrology and structures
}

\author{
Bingru Yang, David Ross-Pinnock, Jody Muelaner, and Glen Mullineux* \\ Department of Mechanical Engineering, University of Bath, Bath BA2 7AY, United Kingdom
}

Received: 25 July 2016 / Accepted: 25 January 2017

\begin{abstract}
Ideally metrology is undertaken in well-defined ambient conditions. However, in the case of the assembly of large aerospace structures, for example, measurement often takes place in large uncontrolled production environments, and this leads to thermal distortion of the measurand. As a result, forms of thermal (and other) compensation are applied to try to produce what the results would have been under ideal conditions. The accuracy obtained from current metrology now means that traditional compensation schemes are no longer useful. The use of finite element analysis is proposed as an improved means for undertaking thermal compensation. This leads to a "hybrid approach" in which the nominal and measured geometry are handled together. The approach is illustrated with a case study example.
\end{abstract}

Keywords: thermal compensation / large volume metrology / finite element analysis / assembly

\section{Introduction}

Assembly operations for large volume products as in the aerospace, marine, and automotive industries are predominantly carried out in large buildings with complex temperature distributions. Thermal variations of several degrees can often be measured across both vertical and horizontal distances. Over a $24 \mathrm{~h}$ period, changes in temperature can reach in excess of $15^{\circ} \mathrm{C}$. Depending upon the heating system of the space, localised temperature loads can also be present.

The standard temperature for metrology activities is recommended to be a uniform $20^{\circ} \mathrm{C}$ [1]. But in such large assembly areas, it is often impractical or costly to closely control the temperature. This means that uncertainty due to thermal expansion and other effects is often the largest contributor to dimensional measurement uncertainty. The problem is made worse since materials commonly used in such assemblies often have high coefficients of thermal expansion.

Practitioners and instrument vendors in large volume metrology have been aware of these limitations for some time [2-4]. The laser tracker is one of the most widely used instruments for coordinate measurement over several tens of metres. Commercially available laser trackers are often equipped with a "weather station" that continuously logs the temperature at the instrument. This means that software can be used to scale measurements which are taken. One approach is to use the coefficient of thermal expansion (CTE) of the material of the measurand. This

\footnotetext{
* Corresponding author: g.mullineux@bath.ac.uk
}

makes many assumptions. It supposes that the temperature of the measurand is uniform and the same as that of the measuring instrument: when dealing with large volumes this is seldom the case. Even if uniformity can be assumed, measurements at non-standard temperatures can be problematic [5].

What is required is a means for compensating for the fact that the ambient conditions in the measurement environment are far from perfect. One area which has been extensively investigated is compensation for thermal effects in machine tools [6-8]. This is a more difficult problem since temperature changes may arise not only from the ambient conditions but also from heat sources within the machine itself. The common approach is to model the physical system using finite element analysis (FEA) and hence predict the resultant deformations.

The aim of this paper is to explore a "hybrid approach" to metrology $[9,10]$ in which measurements of a component (in non-ideal conditions) are used hand-in-hand with a nominal geometry model of that component. There is then a need to be able to map between measurement space and the nominal space. It is proposed that FEA can be used to achieve this. There is currently the ability to measure components with less uncertainty than ever before. However this increased accuracy of the measurements gives no advantage if the part being measured is itself highly distorted due to thermal expansion. The significance of the proposed approach is that it provides the means for making such allowance without introducing significant additional uncertainty.

The next section considers the ideas of thermal compensation. Potential techniques range from simple linear scaling through to the use of analysis software. This 


\begin{tabular}{|c|c|c|c|c|}
\hline \multirow{2}{*}{$\begin{array}{c}\text { Nominal } \\
\text { design space }\end{array}$} & Manufacture & \multirow{2}{*}{$\begin{array}{c}\text { Standard } \\
\text { environment }\end{array}$} & $\overrightarrow{\text { Transform }}$ & \multirow{2}{*}{ Measurement } \\
\hline & & & & \\
\hline
\end{tabular}

Fig. 1. Nominal and measurement spaces.

leads into the use of the hybrid approach and FEA. While FEA can be a highly precise modelling tool, its accuracy depends upon the input data (parameters) it is given. An issue that arises is the trade-off necessary in practice between rapid processing of components and the need to set the parameters correctly. It is not clear that this has been investigated in any great detail. Section 3 considers a case study in which the thermal distortion of a simple component is modelled using FEA with default boundary conditions. It is found that good agreement with measured results is obtained (significantly better than the traditional use of the CTE) which suggests that choice of parameters may not be a very significant issue.

\section{Thermal compensation}

Perhaps the major challenge of dimensional metrology is that one is always working at the wrong temperature. It is desirable to measure components (and assemblies) at the standard temperature of $20^{\circ} \mathrm{C}$, but it is not always practical so to do. This is particularly the case with large structures (such as aircraft parts) whose environment is an assembly shop with poor control of temperature (and other conditions). As a result, it is widely understood that thermal error is one of the dominant error sources in dimensional metrology.

Effectively there are three spaces in which decisions are being made (cf. Fig. 1). One is the space in which the component is designed (with nominal dimensions). Another is the "standard" environment is which measurement ideally would take place (for example at a temperature of $20^{\circ} \mathrm{C}$ ) and for which the nominal dimensional are specified. The third is the nominal space and measurement environment in which measurement is actually carried (for example in factory with poorly controlled temperature). The move from design to the measurement space occurs when the component is manufactured. There is a need to be able to move to and fro between the two measurement environments so that results about one of them can inform decisions made about the other. This paper is concerned with how the transform between the two measurement environments can be achieved.

One approach to metrology is to argue that measurement can take place in the measurement environment provided that appropriate compensation (the transform) is applied to bring the results to the standard environment. An alternative view is that standard values can be transformed into the measurement environment so that appropriate decisions can be made there.

There are a number of methods for dealing with the transform between measurement environments. One which is commonly used in industry to compensate thermal effects [2] is known as "linear scaling" based upon the CTE $\alpha$ of the material of the measurand. Here the amount of expansion or contraction is calculated based on the difference $\Delta T$ between the required nominal temperature and the averaged temperature across the measurement environment or a period of time. The transform is then based on applying an appropriate scale factor to the dimensions in one space to arrive at the other. This scale factor is $1+\alpha(\Delta T)$ when passing from the standard environment to the measurement environment.

However, there are drawbacks. The CTE is not always well-defined. It can vary between samples of the same material, and it can vary even across a component of a single material. The uncertainty of the values of the coefficient combines with the uncertainties in measuring dimensional values and temperature to increase the overall uncertainty of the measuring process.

An alternative method is not to deal with the CTE per se, but instead to consider the results of measuring positions on the physical model and considering the corresponding points on the nominal model, which is usually provided as some form of geometric (computer aided design - CAD) model. A geometric transform is then sought to map between these two sets of points. This can be taken to be the combination of a rigid-body transform and an overall scale factor. This process is commonly known as finding a "7-parameter best fit transformation" [11]. The scale factor that is found effectively takes the place of the CTE.

The approach assumes that thermal effects are homogeneous. However, in many cases, thermal errors can be significantly different in different directions and rotations [5].

A refinement of the approach is to consider the transform required for different sub-parts of features of a measurand. Separate transforms, derived for each of these, can be interpolated across the entire measurand to form an overall transform [12].

A fourth approach to dealing with the transform is to use FEA to establish a map from the nominal CAD model to the measured component. This involves using the CAD data as the basis for a mesh model of the measurand. The effects of changes in temperature are simulated using the FEA. The result is a distorted model which "predicts" what happens in measurement space.

There are usually two stages to the solution process for FEA. The first uses data for temperature at various measured points (on the measurand) to establish the temperature at every node on the mesh model. The second stage is to use this complete set of nodal temperatures to determine the deformation of the measurand. Since the first stage may need to be iterative to deal with variations in temperatures over a time period, it may require a large amount of processing.

What emerges from the above discussion is that a CAD model is often used as the basis for the nominal model, while the measurand itself acts to define measurement space. This has led to the proposal of a hybrid approach $[9,10]$ as suggested in Figure 2. In the upper part of the figure, the nominal geometry (provided by the CAD model) is used to 


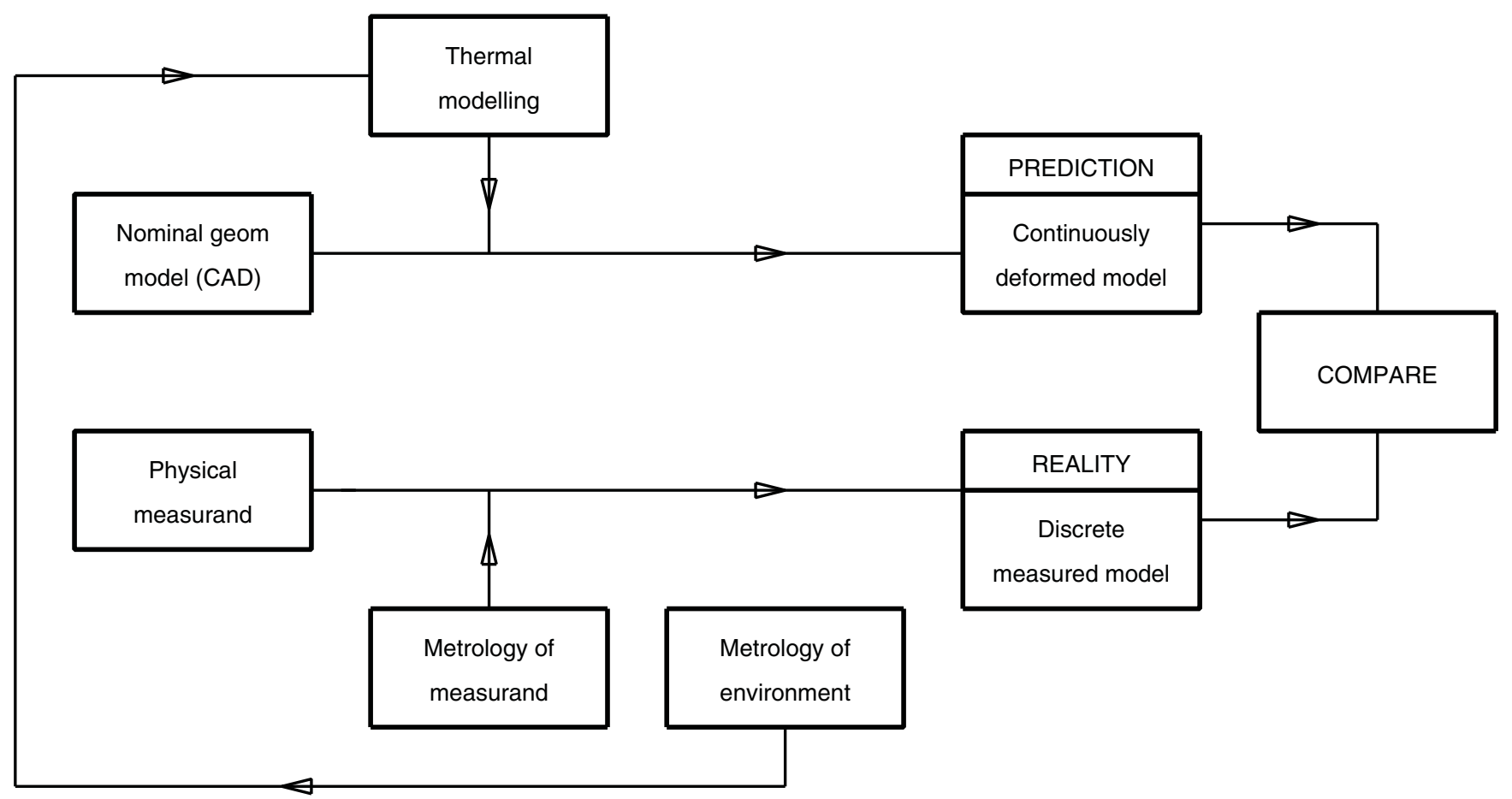

Fig. 2. Hybrid approach to dimensional metrology.

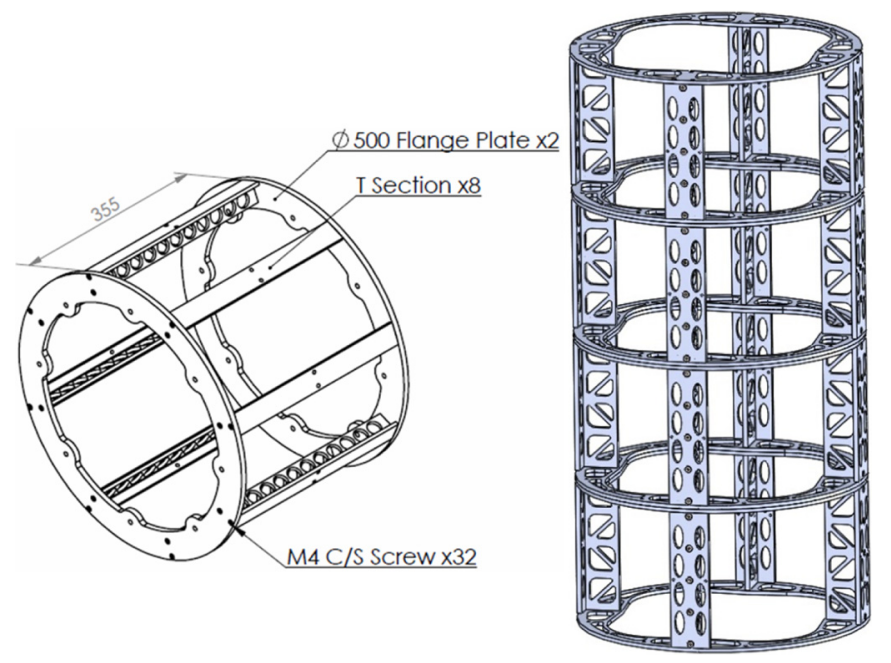

Fig. 3. Design of assembly structure.

make predictions about how deformations occur. In the lower part, metrology techniques are used to inspect the measurand. The results from the two parts can then be compared and conclusions drawn.

A number of problems exist. As more variables controlling the inspection process are taken into account so there is more uncertainty introduced into the results. These variables may be difficult to control and may require to the user to have a good understanding of their implications.

While FEA systems can potentially give very precise results, their accuracy depends upon the quality of the input data. As more parameters are introduced, it is more difficult to ensure that the data is good. For example,

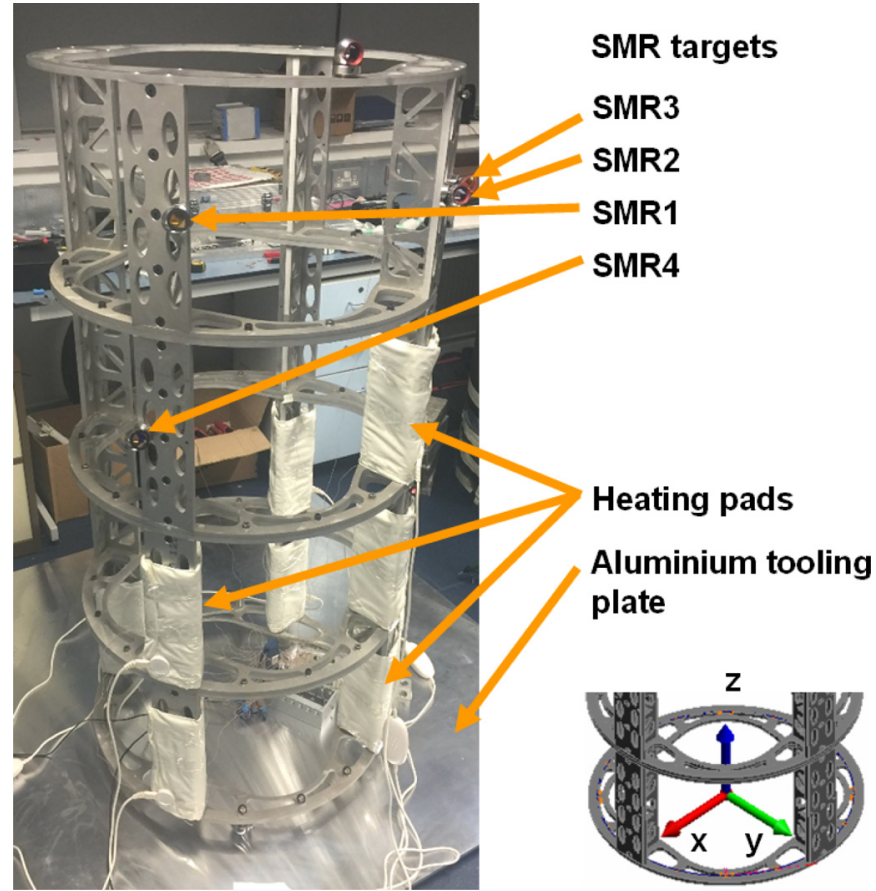

Fig. 4. Experimental set-up.

when a thermal analysis is performed, the FEA software needs to make use of boundary conditions concerning heat flow in terms of radiation and convection. However, it is often not clear how the relevant values should be set. It has been suggested that extensive preliminary experimentation is required with a FEA model in order to ensure good understanding and specification of parameters [13]. 


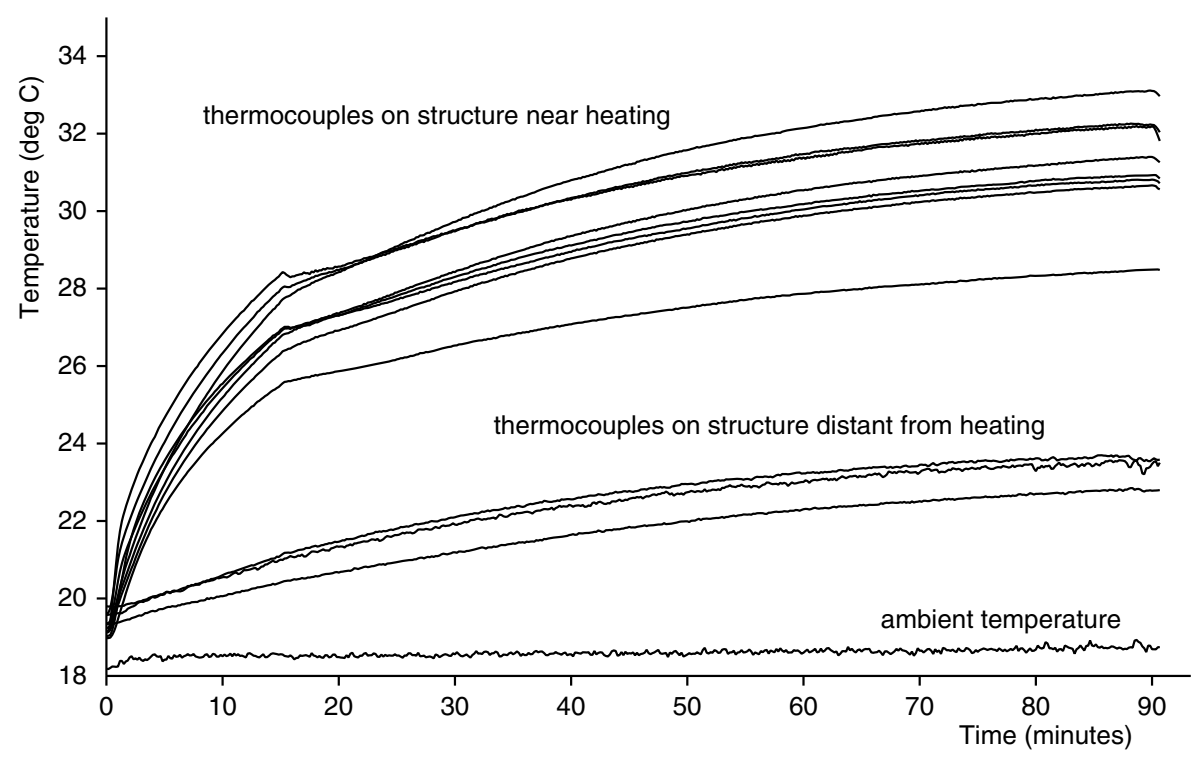

Fig. 5. Heating profile.
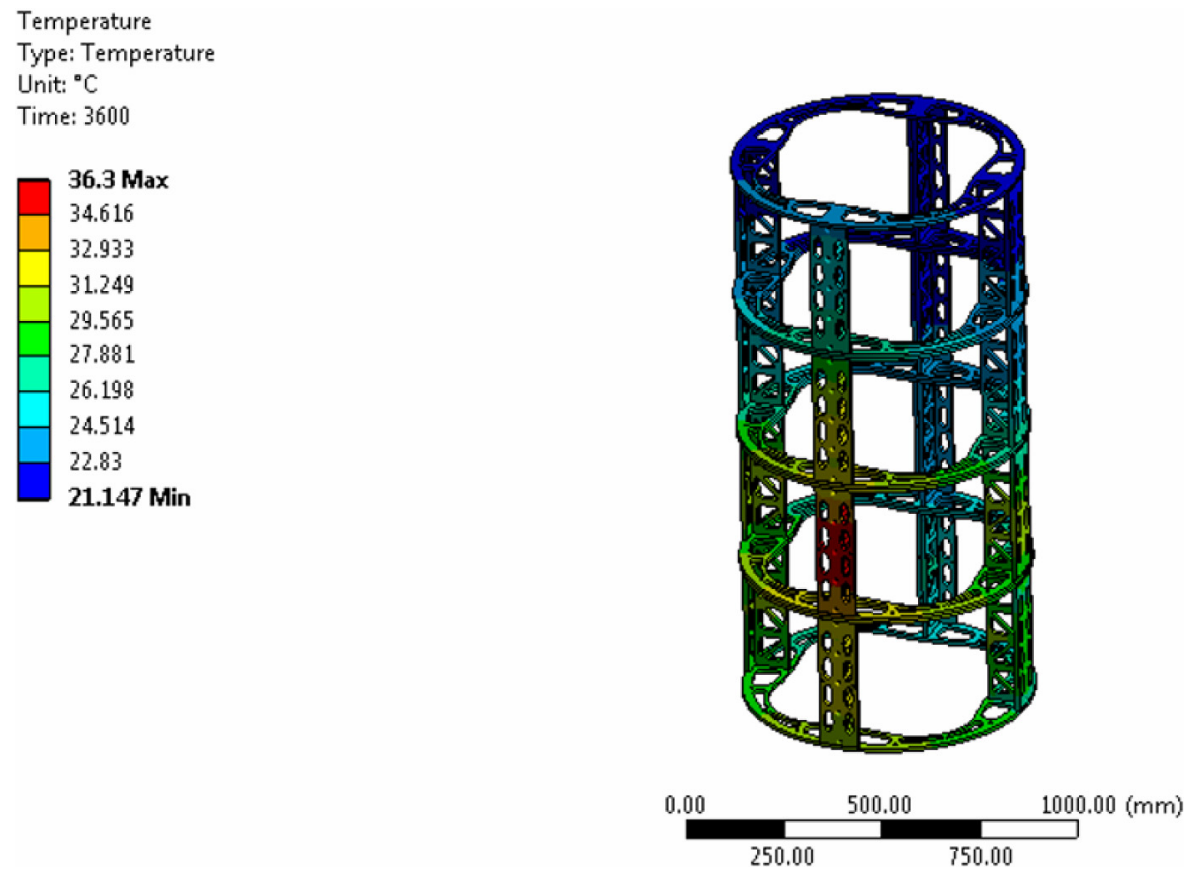

Fig. 6. Transient thermal analysis.

In practice, the possibility of undertaking extensive preliminary work may not exist. It is often desired to be able to take appropriate measurements of a component (and its environment) and immediately deduce the required compensation. It seems to be an unresolved question as to how accurate or effective such an approach can be. To try to obtain some insight into possible magnitudes of errors, the next section considers the measurement of a simple component. Thermal effects are modelled using default values for boundary conditions, and the experimental and analysis results are compared.

\section{Case study example}

The example is based around an assembled structure created to represent a simplified aircraft fuselage. It consists of four identical sub-assemblies. Each sub-assembly is formed from two flange plates and eight T-sections. These are manufactured from aluminium alloy. They are bolted together to create the cylindrical form shown on the left in Figure 3 with a diameter of $750 \mathrm{~mm}$ and a height of $355 \mathrm{~mm}$. The assembled structure formed from the four subassemblies has a height of $1420 \mathrm{~mm}$ and is shown on the right of the figure. 

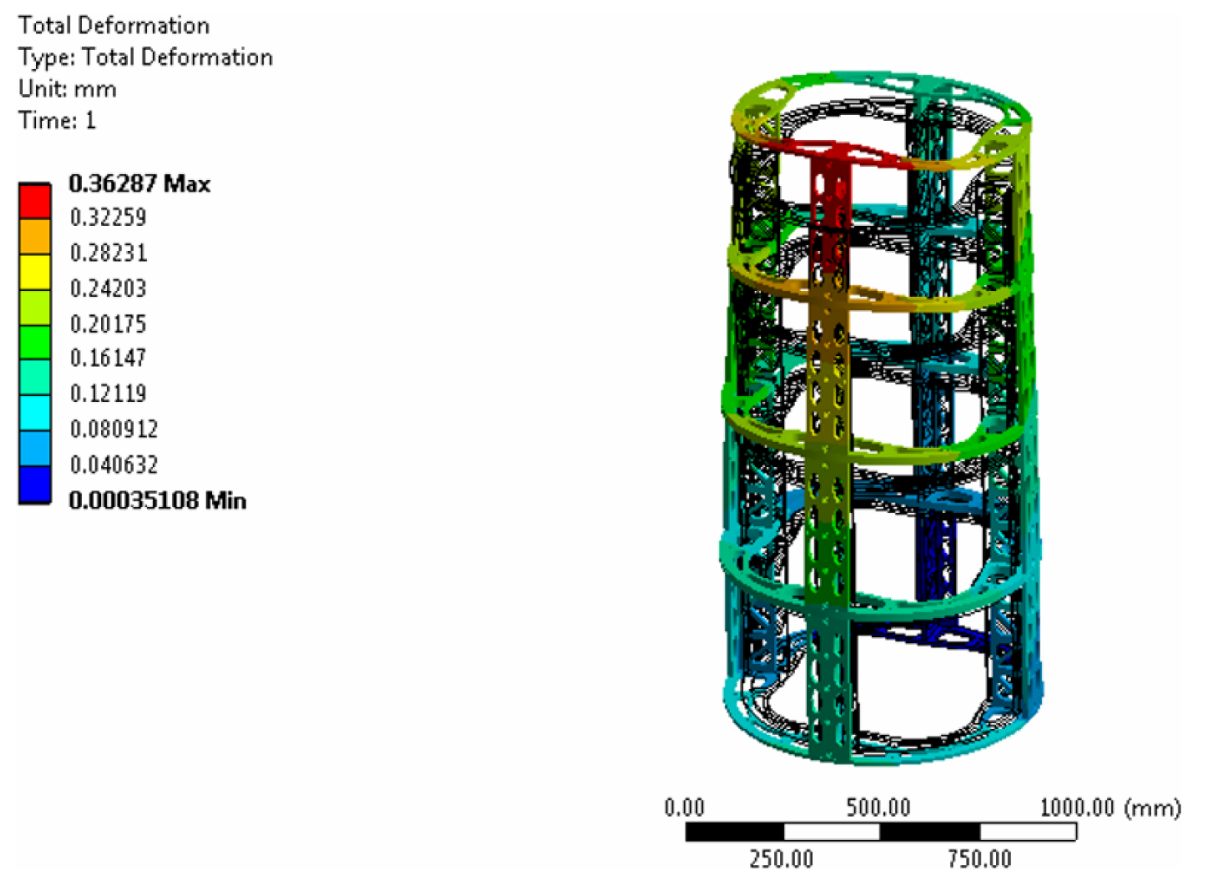

Fig. 7. Structural analysis.

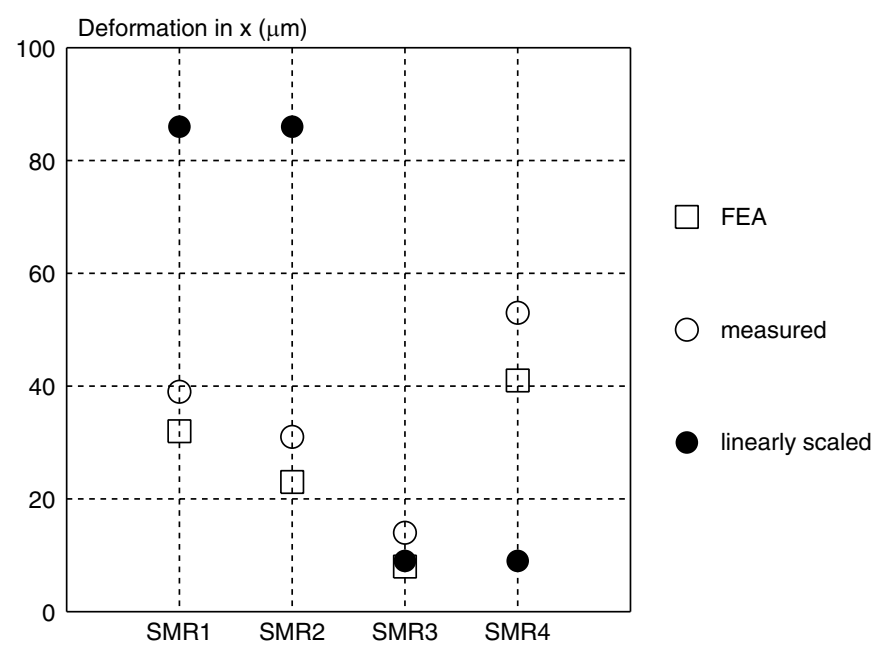

Fig. 8. Comparison of $x$-deformations.

The interest was in the effects of localized heating. To achieve this, eight heating pads were wrapped around parts of the structure as shown in Figure 4. Each heating pad had a contact surface area of $400 \mathrm{~mm} \times 300 \mathrm{~mm}$. The layout of the heating pads was chosen to generate non-symmetric thermal expansion on the assembly and hence to induce bending and twisting.

The assembly was supported on an aluminium tooling plate using three tooling balls with a kinematic $(3-2-1)$ mounting configuration $[14,15]$. This means that one point (at the base of the structure) is fixed and the rest of the structure can move around it. A second point (again on the base) is constrained to move linearly by allowing a tooling ball to move along a channel. A third point (on the base) has its tooling ball constrained to move on a planar metal plate.
The surface temperature was measured at 13 points on the structure using type $\mathrm{T}$ thermocouples. An additional thermocouple was used to monitor the ambient temperature.

The deformation at four points was measured during the heating process using a laser tracker. Spherically mounted retro-reflectors (SMRs) were fixed at four points on the structure at suitably chosen surface and hole positions.

The heating process lasted $90 \mathrm{~min}$. Rapid heating took place during the first $30 \mathrm{~min}$ with the rate reducing after this. This resulted in a temperature variation across the structure (top to bottom) of approximately $10^{\circ} \mathrm{C}$. Temperatures were logged every minute and the deformation of the structure was measured using the SMRs every $15 \mathrm{~min}$. The heating profile recorded by the thermocouples is shown in Figure 5.

To model the heating process appropriately using FEA, a coupled thermal-structural model was created in ANSYS [16]. This involved two stages. The first was a transient thermal analysis used to simulate and determine the thermal distribution across the structure under the given heating condition. This was based on the use of the measured temperatures. Since the rate of heat input varied, it was necessary to perform the transient analysis by timestepping and this meant that the this stage took several minutes to complete its calculations. The default settings for the software for boundary conditions (such as convection and radiation) were assumed.

The results of the thermal analysis were temperature values at each of the finite element nodes. In the second stage, these nodal temperatures were input to a static structural ANSYS model to calculate the deformation of the assembly under thermal loadings. Since this was a oneoff calculation, the calculation time was less than $2 \mathrm{~min}$. 


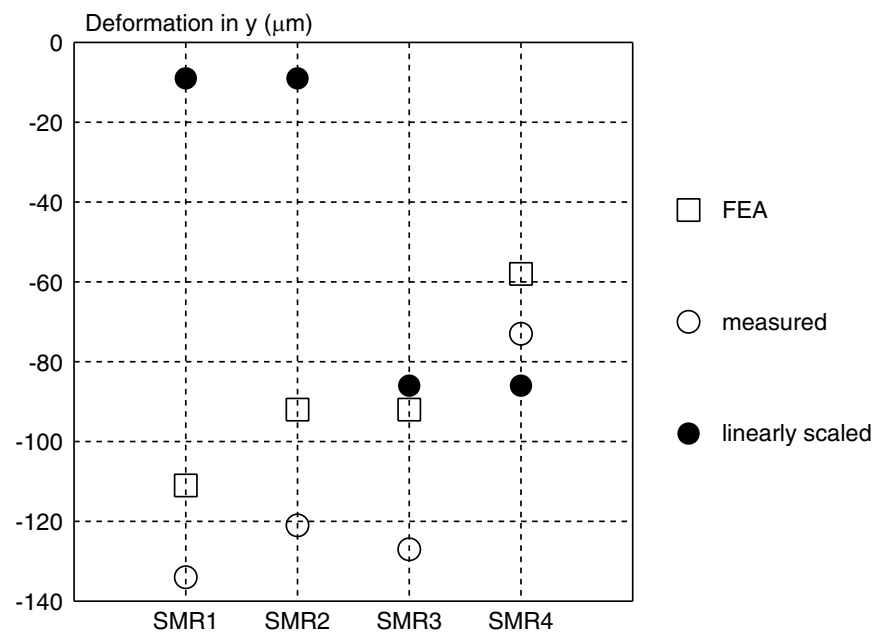

Fig. 9. Comparison of $y$-deformations.

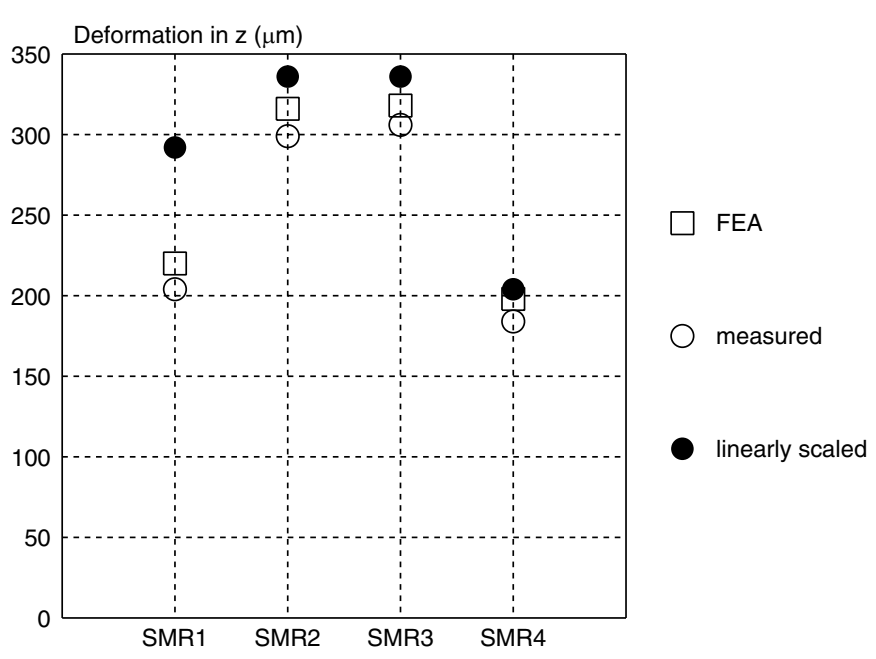

Fig. 10. Comparison of $z$-deformations.

Table 1. Means and uncertainties of SMR measurements at $90 \mathrm{~min}$.

\begin{tabular}{|c|c|c|c|c|c|c|c|c|}
\hline & \multicolumn{2}{|c|}{ SMR 1} & \multicolumn{2}{|c|}{ SMR 2} & \multicolumn{2}{|c|}{ SMR 3} & \multicolumn{2}{|c|}{ SMR 4} \\
\hline & $\begin{array}{l}\text { Mean } \\
(\mathrm{mm})\end{array}$ & $\begin{array}{l}\text { Uncertainty } \\
(\mathrm{mm})\end{array}$ & $\begin{array}{l}\text { Mean } \\
(\mathrm{mm})\end{array}$ & $\begin{array}{l}\text { Uncertainty } \\
(\mathrm{mm})\end{array}$ & $\begin{array}{l}\text { Mean } \\
(\mathrm{mm})\end{array}$ & $\begin{array}{l}\text { Uncertainty } \\
(\mathrm{mm})\end{array}$ & $\begin{array}{l}\text { Mean } \\
(\mathrm{mm})\end{array}$ & $\begin{array}{l}\text { Uncertainty } \\
(\mathrm{mm})\end{array}$ \\
\hline$X$ disp & 0.039 & \pm 0.024 & 0.031 & \pm 0.026 & 0.014 & \pm 0.027 & 0.053 & \pm 0.025 \\
\hline$Y$ disp & 0.134 & \pm 0.025 & -0.121 & \pm 0.024 & -0.127 & \pm 0.025 & -0.073 & \pm 0.025 \\
\hline$Z$ disp & 0.204 & \pm 0.023 & 0.299 & \pm 0.023 & 0.306 & \pm 0.024 & 0.184 & \pm 0.024 \\
\hline
\end{tabular}

Results for thermal and structural analyses are shown in Figures 6 and 7, respectively. Figure 6 shows that the temperature decreases at nodes higher up the structure and that the vertical arm towards the front of the figure is at a higher temperature than the other three. This accords with the expected effects of the heating pads. As a result, the warmer vertical arm deforms to a greater extent than the rest of the structure as is confirmed by Figure 7 .

Results for deformation of the structure are shown in Figures 8-10. These show deformations associated with the four locations of the SMRs: each deformation has been resolved into three mutually perpendicular directions denoted by $x, y, z$ (cf. Fig. 4). The figures give the deformations calculated using the FEA package, and the deformations obtained using the laser tracker.

The SMRs were measured sequentially. When a measurement of an SMR was taken, 1000 values were recorded and averaged so that errors (due to any noise, vibration, etc.) could be eliminated. There was a 5-min gap between each measurement episode and the number of episodes was 19 to cover a time interval of $90 \mathrm{~min}$. As an indication of the characteristics of this process, Table 1 gives values of mean and uncertainty (taken as twice the standard deviation) over the 1000 samples for each SMR at the final measurement episode.

Additionally, the figures show the deformations predicted using linearly scaling based on the CTE. This is obtained as follows. For each measurement position $\mathrm{P}$, the vector from the fixed origin $\mathrm{O}$ of the mounting plate was formed. This was increased in length by the appropriate factor and the new position of $\mathrm{P}$ (relative to $\mathrm{O}$ ) was obtained.

As expected from the heating regime, the major expansion of the structure was in the $z$-direction. There is good agreement between the measured deformation and that obtained using FEA. The "linearly scaled" values also give some agreement in the $z$-direction; consistently they seem to over-estimate the expansion.

The results for the $x$ - and $y$-directions suggest that bending is induced by the heating. In both directions, the FEA shows somewhat less close agreement with the measured deformations, and it consistently underestimates the values. However, it arguably still provides a good estimate of the measured results. However, the linear scaling method fails to produce any agreement with the measurements (or the FEA values).

\section{Conclusions}

There is a dichotomy between the need to undertake metrology at a standard temperature and the ability to be able to do this. This is particularly true when dealing with large structure such as parts of aircraft. As a result various forms of compensation are adopted: the component is measured in non-ideal conditions and the results modified to correspond to ideal ones. Effectively one is working in three spaces, the nominal design space, the 
physical standard environment and the measurement environment, and the compensation scheme provides a map between these.

The state of the art in metrology, driven by increasingly demanding product specifications, requires more sophisticated compensation methods than those traditionally used (such as linear scaling based on the CTE of the material).

What is proposed is the use of FEA. This can be used to model distortion away from the situation at standard temperature, and hence can convert measurements taken at other ambient temperatures to the standard if this is required. There are however challenges involved. There is a need to understand the various parameters involved in a FEA model and to be able to set these appropriately. This may require some experimental work to be undertaken to investigate appropriate values. There may be time constraints on whether such work can be undertaken and there is potentially a trade-off between ease of use and accuracy.

A simple case study example has been presented to illustrate the ideas. Here FEA has been used to model distortions of a component due to heating. Default values were used for the various boundary conditions. Good agreement was achieved between the analysis results and those obtained by measurement. The agreement was significantly better than that achieved with the use of linear scaling, thus indicating the need for an improved approach to thermal compensation. That good agreement was achieved may also suggest that the setting of FEA parameters is not too great an issue.

This work was funded by the EMRP Project IND53. The EMRP is funded by the EMRP participating countries within EURAMET and the European Union. The authors gratefully acknowledge this support and the help and encouragement of all those involved.

\section{References}

1. BSI, BS EN ISO 1: Geometrical Product Specifications (GPS): Standard Reference Temperature for the Specification of Geometrical and Dimensional Properties (BSI, London, 2015)

2. J. Bryan, CIRP Ann. Manuf. Technol. 39, 645 (1990)

3. W.T. Estler, K.L. Edmundson, G.N. Peggs, D.H. Parker, CIRP Ann. Manuf. Technol. 51, 587 (2002)

4. P.G. Maropoulos, J.M. Muelaner, M.D. Summers, O.C. Martin, Int. J. Adv. Manuf. Technol. 70, 621 (2014)

5. D.A. Swyt, J. Res. Natl. Inst. Stand. Technol. 99, 31 (1994)

6. D.G. Ford, S.R. Postlethwaite, J.P. Allen, M.D. Blake, Proc. Inst. Mech. Eng. B: J. Eng. Manuf. 214, 221 (2000)

7. R. Ramesh, M.A. Mannan, A.N. Poo, Int. J. Mach. Tools Manuf. 43, 391 (2003)

8. E. Gomez-Acedo, A. Olarra, M. Zubieta, G. Kortaberria, E. Ariznababarreta, L.N. Lopez de Lacalle, Int. J. Adv. Manuf. Technol. 80, 525 (2015)

9. P.G. Maropoulos, P. Vichare, O. Martin, J. Muelaner, M.D. Summers, A. Kayani, CIRP Ann. Manuf. Technol. 60, 207 (2011)

10. D. Ross-Pinnock, B. Yang, P.G. Maropoulos, in Proc. 38th MATADOR Conference (National Formosa University, Taiwan, 2015), p. 471

11. A. Hashemi, M. Kalantari, M. Kasser, Direct solution of the 7 parameters transformation problem, Appl. Math. Inf. Sci. 7, 1375 (2013)

12. D. Ross-Pinnock, B. Yang, G. Mullineux, Tools and Methods of Competitive Engineering (Delft University of Technology, Delft, The Netherlands, 2016), p. 117

13. N.S. Mian, S. Fletcher, A.P. Longstaff, A. Myers, Meas. Sci. Technol. 22, 085107:1 (2011)

14. C.H. Schouten, P.C.J.N. Rosielle, P.H.J. Schellekens, Precis. Eng. 20, 46 (1997)

15. A. Slocum, Int. J. Mach. Tools Manuf. 50, 310 (2010)

16. ANSYS, ANSYS Simulation Products, Available at: http:// www.ansys.com/Products (last consulted on: July 2016)

Cite this article as: Bingru Yang, David Ross-Pinnock, Jody Muelaner, Glen Mullineux, Thermal compensation for large volume metrology and structures, Int. J. Metrol. Qual. Eng. 8, 21 (2017) 